\title{
Search for contact interactions in inclusive $e p$ scattering at HERA
}

\section{Ivan Pidhurskyi, Mykyta Shchedrolosiev}

Taras Shevchenko National University

E-mail: ivan.pidhurskyi@desy.de, mykyta.shchedrolosievedesy.de

\section{Oleksii Turkot*, Katarzyna Wichmann}

DESY

E-mail: oleksii.turkotedesy.de, katarzyna.wichmann@desy.de

\section{Aleksander Filip Żarnecki}

University of Warsaw

E-mail: Filip.Zarnecki@fuw.edu.pl

\section{on behalf of the ZEUS collaboration}

\begin{abstract}
The high-precision HERA data are used to search for Beyond the Standard Model contributions to electron-quark scattering in the framework of eeqq contact interactions (CI). Combined measurements of the inclusive deep inelastic cross sections in neutral and charged current $e p$ scattering are considered, corresponding to a luminosity of around $1 \mathrm{fb}^{-1}$. The analysis of the inclusive $e p$ data is based on the simultaneous fits of parton distribution functions together with contributions of CI couplings to ep scattering. Results are presented for different general CI scenarios and heavy leptoquarks with expected $95 \%$ C.L. limits on the CI mass scales in the $10 \mathrm{TeV}$ range.
\end{abstract}

The 39th International Conference on High Energy Physics (ICHEP2018)

4-11 July, 2018

Seoul, Korea

${ }^{*}$ Speaker. 


\section{Introduction}

The ZEUS collaboration has used the HERA combined measurement of inclusive cross sections [1] to set limits on possible deviations from the Standard Model due to a finite radius of the quarks [2]. A new approach to the beyond the Standard Model analysis was used, based on the simultaneous fits of parton distribution functions together with contributions of "new physics" processes. This is the only method to properly take into account the possibility that the PDF set may already have been biased by partially or totally absorbing previously unrecognised BSM contributions. The details of this procedure are described in [2]. In the analysis presented here, the new procedure to set limits on the BSM model contributions is applied to other CI scenarios. This work supersedes the results presented in [3] and extends the analysis presented there to more scenarios.

\section{Models for new physics}

Four-fermion contact interactions (CI) represent an effective theory which describes lowenergy effects due to physics at much higher energy scales. The CI approach is not renormalizable and is only valid in the low-energy limit. Vector contact interaction currents considered here are represented by additional terms in the Standard Model Lagrangian:

$$
\mathscr{L}_{C I}=\sum_{\substack{i, j=L, R \\ q=u, d, c, c, b}} \eta_{i j}^{e q}\left(\bar{e}_{i} \gamma^{\mu} e_{i}\right)\left(\bar{q}_{j} \gamma_{\mu} q_{j}\right)
$$

where the sum runs over electron and quark helicities and quark flavors. The couplings $\eta_{i j}^{e q}$ describe the helicity and flavor structure of contact interactions.

For the general contact interaction scenarios considered here, it was assumed that all up-type quarks have the same contact-interaction couplings, and a similar assumption was made for downtype quarks, leading to eight independent couplings, $\eta_{i j}^{e q}$, with $q=u, d$. A set of one-parameter scenarios were defined by eight coefficients, $\varepsilon_{i j}^{e q}$, each of which may take the values \pm 1 or zero, see Table 1, and the coupling strength $\eta$. The couplings are given by the formula:

$$
\eta_{i j}^{e q}=\varepsilon_{i j}^{e q} \eta=\varepsilon_{i j}^{e q} \frac{4 \pi}{\Lambda^{2}} .
$$

Leptoquarks (LQ) appear in certain extensions of the SM that connect leptons and quarks; they carry both lepton and baryon numbers and have spin 0 or 1 . According to the general classification proposed by Buchmüller, Rückl and Wyler [4], there are 14 possible LQ states: seven scalar and seven vector . In the limit of heavy LQs $\left(M_{L Q} \gg \sqrt{s}\right)$, the effect of $s$ - and $t$-channel LQ exchange is equivalent to a vector-type eeqq contact interaction ${ }^{1}$. The effective $\mathrm{LQ}$ coupling, $\eta_{\mathrm{LQ}}$, is given by the square of the ratio of the leptoquark Yukawa coupling, $\lambda_{L Q}$, to the leptoquark mass, $M_{L Q}$, and the contact-interaction couplings of the Lagrangian (2.1), $\eta_{i j}^{e q}$, can be then written as:

$$
\eta_{i j}^{e q}=a_{i j}^{e q} \cdot \eta_{\mathrm{LQ}}=a_{i j}^{e q}\left(\frac{\lambda_{L Q}}{M_{L Q}}\right)^{2}
$$

where the coefficients $a_{i j}^{e q}$ depend on the LQ species and are twice as large for vector as for scalar leptoquarks.

\footnotetext{
${ }^{1}$ For the invariant mass range accessible at HERA, $\sqrt{s} \sim 300 \mathrm{GeV}$, heavy LQ approximation is applicable for $M_{L Q}>400 \mathrm{GeV}$.
} 
HERA $e^{ \pm} p$ 1994-2007 data

\begin{tabular}{|c|c|c|c|c|c|c|}
\hline \multirow{2}{*}{\multicolumn{2}{|c|}{ Coupling structure }} & \multicolumn{4}{|c|}{ 95\% C.L. limits (TeV) } & \multirow{3}{*}{$\begin{array}{l}p_{S M} \\
(\%)\end{array}$} \\
\hline & & \multicolumn{2}{|c|}{ Measured } & \multicolumn{2}{|c|}{ Expected } & \\
\hline Model & {$\left[\varepsilon_{L L}, \varepsilon_{L R}, \varepsilon_{R L}, \varepsilon_{R R}\right]$} & $\Lambda^{-}$ & $\Lambda^{+}$ & $\Lambda^{-}$ & $\Lambda^{+}$ & \\
\hline $\mathrm{LL}$ & {$[+1, \quad 0, \quad 0,0]$} & 18.9 & 4.5 & 5.9 & 6.3 & 6.6 \\
\hline $\mathrm{RR}$ & {$\left[\begin{array}{lll}0, & 0, & 0,+1\end{array}\right]$} & 27.2 & 4.4 & 5.7 & 6.1 & 5.5 \\
\hline LR & {$\left[\begin{array}{llll}0, & +1, & 0, & 0\end{array}\right]$} & 5.0 & 7.1 & 5.7 & 6.3 & 34 \\
\hline RL & {$\left[\begin{array}{llll}0, & 0,+1, & 0\end{array}\right]$} & 5.4 & 6.3 & 5.6 & 6.5 & 41 \\
\hline VV & {$[+1,+1,+1,+1]$} & 14.7 & 9.7 & 11.2 & 11.4 & 26 \\
\hline AA & {$[+1,-1,-1,+1]$} & - & $5.0-11.1$ & 7.9 & 7.8 & 0.6 \\
\hline VA & {$[+1,-1,+1,-1]$} & $\begin{array}{c}3.76 \\
-\end{array}$ & $3.6-10.2$ & 4.2 & 4.2 & $\begin{array}{l}5.8 \\
2.8\end{array}$ \\
\hline $\mathrm{X} 1$ & {$[+1,-1, \quad 0,0]$} & - & $3.5-6.8$ & 5.4 & 5.5 & 0.4 \\
\hline $\mathrm{X} 2$ & {$[+1, \quad 0,+1,0]$} & 10.1 & 6.9 & 7.8 & 8.3 & 24 \\
\hline $\mathrm{X} 3$ & {$[+1, \quad 0, \quad 0,+1]$} & 24.4 & 6.3 & 8.3 & 8.7 & 6.7 \\
\hline $\mathrm{X} 4$ & {$[0,+1,+1,0]$} & 7.4 & 9.4 & 8.0 & 8.6 & 38 \\
\hline $\mathrm{X} 5$ & {$\left[\begin{array}{lll}0, & +1, & 0,+1\end{array}\right]$} & 10.1 & 6.9 & 7.7 & 7.7 & 27 \\
\hline X6 & {$[0,0,+1,-1]$} & $3.4-6.0$ & - & 5.3 & 5.5 & 0.3 \\
\hline
\end{tabular}

Table 1: Relations between couplings $\left[\varepsilon_{L L}, \varepsilon_{L R}, \varepsilon_{R L}, \varepsilon_{R R}\right]$ for the compositeness models and the upper 95\% C.L. limits on the compositeness scale, $\Lambda$, corresponding to the central $90 \%$ C.L. intervals on the CI coupling obtained from the ZEUS analysis of the HERA inclusive data. Also shown are the expected limits, and the probability of SM to result in the best-fit coupling value greater than that observed for the data, $p_{S M} . \Lambda^{+}$and $\Lambda^{-}$limits correspond to the scenarios with positive and negative coupling values, respectively. The same coupling structure applies to all quarks. For AA, VA, X1 and X6 models, ranges of compositeness scale values corresponding to the central $90 \%$ C.L. coupling interval are given.

\section{Results}

The $95 \%$ C.L. limits on the compositeness scale $\Lambda$ for different CI models are summarized in Table 1. For most of the considered CI scenarios improved description of the HERA data is obtained either for positive or for negative coupling values. Only in case of the VA model two minima are observed in the $\chi^{2}$ dependence on coupling value, one for positive and one for negative couplings. As different coupling signs correspond to distinct scenarios, we calculate two confidence intervals for the VA model, separately for negative and positive couplings.

The limits on the ratio of the Yukawa coupling to the leptoquark mass, $\lambda_{L Q} / M_{L Q}$, corresponding to the central $90 \%$ C.L. coupling intervals, are summarized in Table 2 together with the coefficients $a_{i j}^{e q}$ describing the CI coupling structure. For two models, $S_{1}^{L}$ and $V_{\circ}^{R}$, significant improvement in the description of HERA data can be obtained and probability of reproducing the fit result with Standard Model replicas, $p_{S M}$, is below 5\%, which means that $\eta_{\mathrm{LQ}}=0$ (corresponding to the Standard Model) is outside the central 90\% C.L. interval. For both models the range of $\lambda_{L Q} / M_{L Q}$ values is given, corresponding to the central 90\% C.L. $\eta_{\mathrm{LQ}}$ interval. Also for $\tilde{S}_{\circ}^{R}$ and $V_{\circ}^{L}$ models ${ }^{2}$

\footnotetext{
${ }^{2}$ Please note that $\tilde{S}_{\circ}^{R}$ is related to $V_{\circ}^{R}$ model, corresponding to the same CI coupling structure, but with different sign.
} 


\begin{tabular}{|cl|c|c|c|}
\multicolumn{5}{|c|}{ HERA $e^{ \pm} p 1994-2007$ data } \\
\hline \multirow{3}{*}{ Model } & Coupling Structure & \multicolumn{2}{|c|}{$\lambda_{L Q} / M_{L Q}\left(\mathrm{TeV}^{-1}\right)$} & \\
\cline { 3 - 4 } & & \multicolumn{2}{|c|}{$95 \%$ C.L. limits } & $p_{S M}$ \\
\cline { 3 - 4 } & & Measured & Expected & $(\%)$ \\
\hline$S_{\circ}^{L}$ & $a_{L L}^{e u}=+\frac{1}{2}$ & 0.27 & 0.56 & 8.4 \\
$S_{\circ}^{R}$ & $a_{R R}^{e u}=+\frac{1}{2}$ & 1.02 & 0.72 & 5.9 \\
$\tilde{S}_{\circ}^{R}$ & $a_{R R}^{e d}=+\frac{1}{2}$ & - & 1.71 & 1.8 \\
$S_{1 / 2}^{L}$ & $a_{L R}^{e u}=-\frac{1}{2}$ & 0.80 & 0.76 & 42 \\
$S_{1 / 2}^{R}$ & $a_{R L}^{e d}=a_{R L}^{e u}=-\frac{1}{2}$ & 0.99 & 0.92 & 37 \\
$\tilde{S}_{1 / 2}^{L}$ & $a_{L R}^{e d}=-\frac{1}{2}$ & 1.51 & 1.39 & 41 \\
$S_{1}^{L}$ & $a_{L L}^{e d}=+1, a_{L L}^{e u}=+\frac{1}{2}$ & $0.78-1.16$ & 0.62 & $<0.01$ \\
\hline$V_{\circ}^{L}$ & $a_{L L}^{e d}=-1$ & - & 0.44 & 0.4 \\
$V_{0}^{R}$ & $a_{R R}^{e d}=-1$ & $0.56-1.44$ & 0.99 & 1.8 \\
$\tilde{V}_{0}^{R}$ & $a_{R R}^{e u}=-1$ & 0.16 & 0.53 & 6.3 \\
$V_{1 / 2}^{L}$ & $a_{L R}^{e d}=+1$ & 1.11 & 1.29 & 38 \\
$V_{1 / 2}^{R}$ & $a_{R L}^{e d}=a_{R L}^{e u}=+1$ & 0.53 & 0.57 & 39 \\
$\tilde{V}_{1 / 2}^{L}$ & $a_{L R}^{e u}=+1$ & 0.47 & 0.49 & 42 \\
$V_{1}^{L}$ & $a_{L L}^{e d}=-1, a_{L L}^{e u}=-2$ & 0.39 & 0.35 & 31 \\
\hline
\end{tabular}

Table 2: Coefficients $a_{i j}^{e q}$ defining the effective leptoquark couplings in the contact-interaction limit, $M_{L Q} \gg$ $\sqrt{s}$, limits on the Yukawa coupling to the leptoquark mass ratio, $\lambda_{L Q} / M_{L Q}$, corresponding to the central 90\% C.L. intervals on the LQ coupling obtained from the presented analysis, and the probability of SM to result in the best-fit coupling value greater than that observed for the data, $p_{S M}$, for different models of scalar (upper part of the table) and vector (lower part) leptoquarks. For $\tilde{S}_{\circ}^{R}$ and $V_{\circ}^{L}$ models limit values are not given as the confidence intervals cover unphysical (negative) coupling values only. For $S_{1}^{L}$ and $V_{0}^{R}$ models ranges of values corresponding to the central $90 \%$ C.L. $\eta_{\mathrm{LQ}}$ intervals is given.

probability below $5 \%$ is obtained, but the confidence interval covers unphysical (negative) coupling values only so limit values are not given.

\section{References}

[1] H1 and ZEUS Collaborations, H. Abramowicz et al., Combination of measurements of inclusive deep inelastic $e \pm p$ scattering cross sections and QCD analysis of HERA data, Eur. Phys. J. C 75 (2015) 1 [arXiv:1506.06042]

[2] ZEUS Collaborations, $\mathrm{H}$. Abramowicz et al., Limits on the effective quark radius from inclusive ep scattering at HERA, Phys. Lett. B 757 (2016) 468 [arXiv: 1604 . 01280]

[3] A. F. Żarnecki, Limits on the effective quark radius from inclusive ep scattering \& contact interactions at HERA, PoS ICHEP2016 (2016) 146 [arXiv: 1611.03825]

[4] W. Buchmüller, R. Rückl and D. Wyler, Leptoquarks in lepton-quark collisions, Phys. Lett. B 191 (1987) 442 [doi: 10.1016/0370-2693 (87) 90637-X] 